Meta

Journal des traducteurs

Translators' Journal

\title{
Comment dénaturer une traduction
}

\section{Maurice Pergnier}

Volume 35, numéro 1, mars 1990

Actes du colloque international " La traduction proligère "

URI : https://id.erudit.org/iderudit/003824ar

DOI : https://doi.org/10.7202/003824ar

Aller au sommaire du numéro

Éditeur(s)

Les Presses de l'Université de Montréal

ISSN

0026-0452 (imprimé)

1492-1421 (numérique)

Découvrir la revue

Citer cet article

Pergnier, M. (1990). Comment dénaturer une traduction. Meta, 35(1), 219-225.

https://doi.org/10.7202/003824ar

Ce document est protégé par la loi sur le droit d'auteur. L’utilisation des services d’Érudit (y compris la reproduction) est assujettie à sa politique d'utilisation que vous pouvez consulter en ligne.

https://apropos.erudit.org/fr/usagers/politique-dutilisation/
Cet article est diffusé et préservé par Érudit.

Érudit est un consortium interuniversitaire sans but lucratif composé de l’Université de Montréal, l’Université Laval et l’Université du Québec à Montréal. Il a pour mission la promotion et la valorisation de la recherche. https://www.erudit.org/fr/ 


\section{COMMENT DÉNATURER UNE TRADUCTION}

MAURICE Pergnier

Université Paris XII-Val-de-Marne, Créteil, France

Il est plus fréquent qu'on s'intéresse aux améliorations apportées à une traduction dans les phases successives de son élaboration (depuis la production du premier jet par le traducteur jusqu'aux relectures effectuées par des tiers) qu'aux détériorations que ces différentes phases peuvent introduire. Cela est aussi plus satisfaisant pour l'esprit. Les interventions qui détériorent les traductions ne sont cependant pas rares, et leur étude est aussi instructive que l'étude des interventions positives. Ces détériorations nous renseignent, autant que les améliorations, sur les critères de qualité des traductions.

Le cas d'espèce que nous présentons ici n'est nullement exceptionnel. De plus en plus souvent, en effet, les traductions - surtout quand elles sont livrées au monde de l'édition - échappent au traducteur et font l'objet de la sollicitude de personnes qui les retravaillent et leur impriment leur marque. Nous ne parlons pas ici de la révision stade déjà franchi au moment dont il est question - mais de la phase que, faute d'un mot français admis par tout le monde, nous appellerons la phase de l'editing. Quand nous parlerons ci-après du résultat produit par le traducteur, nous y inclurons évidemment l'apport de la révision.

Le cas présenté ici est exemplaire en ce qu'il cumule deux types d'interventions sur la traduction: celle de professionnels de l'editing et celle de l'auteur travaillant en relation avec eux. Il s'agit d'un cas de figure encore relativement rare en Europe, mais bien connu des traducteurs canadiens, en raison du bilinguisme qui fait que les auteurs traduits connaissent suffisamment la langue de traduction pour se croire autorisés à intervenir activement sur le texte traduit.

Ces interventions se ramènent grossièrement à deux types de modifications contradictoires que nous allons retrouver dans le cas d'espèce étudié:

L'intervention de l'editor se manifeste souvent par l'introduction de fioritures et de restructurations de phrases destinées à «faire joli» et à donner du piquant à la prose. Cette réécriture de certains passages se fait parfois au grand dam de la fidélité au sens.

À l'inverse, mais avec des résultats identiques, l'intervention conjuguée de l'editor et de l'auteur tend à tirer le texte vers une littéralité que le traducteur avait soigneusement évitée pour mieux servir le texte. Cette tendance à réintroduire des calques là où la traduction était plus exacte part de motivations différentes chez l'auteur et chez l'editor.

L'auteur - qui connait assez la langue de traduction pour ses besoins de communication pratique, mais pas assez pour apprécier les nuances d'un texte écrit dans cette langue - croit que sa pensée est trahie quand il ne retrouve pas dans la traduction un enchaînement de mots parallèle à celui de sa prose. Il reste donc crispé sur la «lettre» au lieu de s'accrocher à l'esprit et exigera qu'on adopte la formulation la plus calquée sur sa formulation originale. Le rôle de l'editor devrait normalement être de faire comprendre à l'auteur les raisons de la distance prise à l'égard de la formulation originale pour mieux servir sa pensée et son style. Au lieu de cela, il tendra à abonder dans le même sens pour deux raisons différentes : 
La première, à laquelle nous nous arrêterons peu, car nous nous intéressons plus aux résultats qu'aux mobiles psycho-sociologiques, est que l'editor justifie d'autant mieux son existence qu'il introduit plus de modifications.

La deuxième est d'ordre méthodologique : contrairement au traducteur, qui a abordé la traduction de chaque passage après une longue méditation de l'ensemble et en étant constamment conscient des liens existant entre le passage traité et son contexte général, l'editor juge le texte phrase par phrase, un œil sur la traduction et un œil sur l'original. Il est donc tout naturellement prêt à adopter la même perspective que l'auteur et à considérer comme fautive toute formulation qui s'éloigne du calque. Il croira souvent de bonne foi que la traduction idiomatique d'un passage est une licence que le traducteur s'est octroyée, alors qu'il s'agissait d'une solution réfléchie au problème textuel traité.

Ce travail sur texte fragmenté est dans doute la source principale des dénaturations apportées aux traductions dans les conditions envisagées ici.

Illustrons sur quelques exemples les effets concrets induits par les causes que nous avons rapidement esquissées. En ce qui concerne le texte, je dirai simplement pour la compréhension des extraits analysés qu'il s'agit d'un livre sur l'histoire du Québec, d'environ 500 pages, publié au Canada d'abord en version originale anglaise, puis en version traduite française.

Nous avons essayé de sélectionner, parmi des centaines, des exemples qui ne nécessitent pas une longue présentation du contexte. Je ne m'arrêterai guère à des exemples qui sont purement et simplement des fautes de français, comme le fait de trouver dans un chapitre qui décrit le régime seigneurial au temps de la Nouvelle-France: «Il contracte moultes obligations (...)»

$\mathrm{Si}$ je les mentionne, ce n'est pas parce qu'elles trahissent les ignorances de l'editor qui les a commises, mais pour ce qu'elles manifestent de sa manière de procéder. L'editor a introduit des barbarismes en voulant «enjoliver» la prose du traducteur, qu'il trouvait sans doute trop plate. Le traducteur avait décrit tout simplement:

Il a l'obligation de (...)

L'original anglais disait: «The seigneur had to (provide and maintain...)»

L'editor a voulu «fleurir» ces phrases banales en introduisant ici un archaïsme.

Il s'agit là de bagatelles à jeter en pâture au grammairien. Plus intéressants sont les cas de modifications aboutissant non seulement à des incorrections mais à des inexactitudes ou obscurités. Ces modifications sont toujours dues à une volonté de calquer davantage la traduction sur l'anglais. Prenons-en quelques exemples:

a) 1. Version publiée: Les habitants accueillent les envahisseurs en leur ouvrant leurs bras et leurs provisions.

2. Version traducteur: Les habitants accueillent les envahisseurs en leur ouvrant les bras et en leur offrant leurs provisions.

3. Original anglais: The local habitants greeted the invaders with open arms and open stores ${ }^{1}$.

La version traducteur n'était guère géniale. Pourquoi la détériorer encore en introduisant un zeugma plus qu'osé ? Il n'y a pas d'autre raison que la volonté de maintenir à tout prix la figure de rhétorique permise par la structure de l'anglais mais qui, transposée telle quelle en français, devient ridicule à cause du fonctionnement des adjectifs possessifs.

b) 1. Version publiée: Avec un tel manque de monnaie, et dans le contexte d'une économie liée à l'exportation de matières premières brutes il ne pouvait y avoir dans la colonie ni volonté, ni possibilité de se lancer dans des entreprises industrielles rentables. 
Le lecteur qui lit avec un tel manque de monnaie comprend : en l'absence de pièces et de petites coupures (en anglais change). Or, comment peut-on parler de monnaie à propos de la description de l'économie et de l'industrie d'un pays?

Le fait est que l'editor a tout simplement introduit le mot «monnaie» comme équivalent de l'anglais money = argent et, de ce fait, rendu la phrase obscure et absurde.

Voici l'original anglais :

With no surplus money in the colony and the economy tied to the exporting of an untransformed material resource, there was neither willingness nor ability to develop local moneymaking ventures.

La version du traducteur était pourtant déjà très littérale, trop même. Qu'on en juge :

En l'absence d'excédent d'argent et avec une économie liée à l'exportation de matières brutes, il ne pouvait y avoir (...)

On aurait pu facilement la bonifier, par exemple, en disant : en l'absence de liquidités. Au lieu de cela, la modification aboutit à occulter complètement le sens de l'énoncé, en éliminant surplus et en faisant croire au lecteur, soit qu'il y avait une pénurie de pièces et de petites coupures pour rendre la monnaie, soit qu'aucune monnaie n'avait cours sur le marché canadien à l'époque décrite.

On ne voit guère non plus ce qu'apporte, au niveau du style comme du sens, l'introduction du «contexte d'une économie»...

c) Dans un autre passage, le traducteur avait écrit: «Ceux d'entre eux [il s'agit d'artisans] qui ouvrent boutique grâce au système de l'apprentissage satisfont, et au-delà, aux besoins de la colonie $(\ldots)$ »

Cette formulation ne plaît pas à l'editor, qui reconstruit la phrase en introduisant selon la façon dont on interprète la phrase - soit une incorrection syntaxique, soit une inexactitude de sens :

Ceux d'entre eux qui ouvrent boutique grâce au système de l'apprentissage suffisent et parfois même dépassent les besoins de la colonie (...)

Peut-on «suffire et dépasser des besoins»? On peut à la rigueur excéder des besoins mais non les dépasser... anglais :

Pour chercher la raison d'être de cette modification il suffit de se reporter au texte

Those who did and acquired a trade by means of the apprenticeship system merely filled and sometimes even overstocked the colony's immediate needs (...)

L'editor cherche à rattacher «besoins» aux deux verbes français de la même façon que «needs» est rattaché aux deux verbes anglais. Peut-être, en outre, ne connaît-il pas la tournure satisfaire à?

Prenons un dernier exemple dans un autre registre. Le texte original comporte une phrase dans laquelle il est dit, dans un raccourci humoristique, que «The wives of the voyageurs ${ }^{2}$ in the towns often opened taverns to profit from the many thirsty soldiers that France sent and the colony produced after 1663 ».

Dans le passage souligné, le traducteur avait écrit, pour essayer de rendre le sens humoristique de thirsty (= amateurs de boissons alcoolisées) : «les épouses des voyageurs ouvrent en ville des tavernes pour tirer profit de la soif des nombreux soldats que la France envoie (...)» 
On ne peut pas dire que cette traduction soit particulièrement audacieuse. On aurait pu, par exemple, proposer: ouvrent des tavernes pour exploiter la soif des nombreux soldats. Elle a été pourtant impitoyablement censurée par l'editor et remplacée par le calque pur et simple: «les épouses des voyageurs ouvrent en ville des tavernes pour tirer profit des nombreux soldats assoiffés que la France envoie...» En conséquence de quoi, on comprend que les soldats ont traversé l'Atlantique sans boire une goutte d'eau et que les dames ouvrant taverne vont faire commerce non des boissons mais des soldats eux-mêmes (un type de traite non répertorié dans les annales de l'histoire des Amériques!)...

Voici maintenant un exemple qui n'aboutit pas à une inexactitude, mais qui manifeste bien la façon dont agit la crispation sur la littéralité.

Dans la suite du paragraphe sur le régime seigneurial en Nouvelle-France, dont nous avons déjà extrait moultes, l'auteur écrit: (...) «the seigneur had to provide and maintain a mill and a manor house, support the parish church (...)»

Version du traducteur : (...) il a l'obligation de pourvoir à la construction et à l'entretien d'un moulin à blé, de tenir feu et lieu, de patronner l'église paroissiale (...)

Version publiée: Il contracte moultes obligations : pourvoir à la construction et l'entretien d'un moulin à blé, construire une maison seigneuriale, patronner l'église paroissiale (...)

La comparaison de ces deux versions demande une explication: le texte anglais parle de réalités françaises a partir de sources françaises; le segment provide and maintain a manor house est une paraphrase pour désigner ce que tous les textes de cette époque et sur cette époque appellent «tenir feu et lieu». S'agissant de droit féodal français, l'anglais n'a évidemment pas de terme équivalent, d'où la nécessité d'une traduction explicative. Le traducteur, conscient de cela, avait réintroduit la formulation française usuelle. L'editor, n'imaginant sans doute pas que le traducteur ait pu se documenter, a probablement cru à une grossière incompréhension et a fait une traduction rétroflexe dans laquelle manor house devient «maison seigneuriale ${ }^{3}$ ». Il élimine ainsi la couleur locale et la couleur du temps, préférant les introduire plus haut avec l'archä̈sme «moult» utilisé avec le bonheur d'expression que l'on a vu. Quant à l'auteur, s'il est pour quelque chose dans cette version finale, c'est qu'il a oublié les sources françaises à partir desquelles il a travaillé.

Cette modification de la version traducteur n'est pas une «trahison», même si elle réduit la portée de l'énoncé. Nous la mentionnons seulement pour ce qu'elle révèle de la démarche générale présidant à la correction du manuscrit.

Donnons enfin un exemple qui ne nécessite pas de commentaire, bien qu'il soit atypique par rapport aux précédents.

Anglais : The French may have had the last laugh (...)

Traducteur : En vérité, les Français auraient pu penser : «rira bien qui rira le dernier» (...)

Editor : En vérité, les Français pourraient bien rire de la situation... (sic)

Pour ne pas allonger démesurément notre exposé, nous arrêterons lằ nos analyses d'exemples isolés pour illustrer maintenant notre propos sur un point concernant tout le texte. Il s'agit de l'usage des temps verbaux.

Situons rapidement la question: on sait qu'en anglais le temps unique du récit est le prétérit. En français, les nuances exprimées par le seul prétérit se répartissent subtilement entre trois temps, selon qu'il s'agit de séquences d'événements ou de descriptions notamment. Ces trois temps sont l'imparfait, le passé simple et le présent. On sait que lorsque le présent est utilisé pour narrer des événements du passé, il est appelé présent historique. C'est dire que les ouvrages d'histoire en font un très large usage. Or, on se souviendra 
que le texte dont il est question ici est un livre d'histoire. Le présent historique devait donc tout naturellement faire son apparition sous la plume du traducteur pénétré de l'esprit de son texte, notamment dans des passages où il s'agissait de narrer avec vivacité une séquence d'événements.

Il ne s'agit pas là, chez le traducteur, de l'application d'un système ni du résultat d'une analyse linguistique. C'est tout simplement le fruit de sa sensibilité en profondeur à ce qu'on appelle communément le «génie de le langue».

Quelle ne fut pas la stupeur du traducteur à la lecture du manuscrit corrigé — qu'on condescendit à lui soumettre - lorsqu'il s'aperçut que tous les présents historiques avaient été systématiquement transformés en passés simples, sans égard pour la concordance avec les autres temps, notamment avec les passés composés. Il en résultait non seulement une narration d'une grande platitude, d'où toute animation était bannie, mais aussi une impression «guindée» accentuée par le fait que les passés simples appelaient des subjonctifs imparfaits souvent très lourds à manier. L'horreur ne s'arrêtait pas là : le pire résidait dans le fait que certains de ces passés simples étaient grossièrement fautifs. Ainsi, un présent historique «ils entrevoient» était devenu rien de moins que le passé simple «ils entrevoyèrent». Ce type de manipulation d'un texte requiert d'une part une très grande maîtrise du système verbal et, d'autre part, un sens aigu de la dynamique des textes; deux compétences dont on se demande si elles étaient vraiment le fort du relecteur investi par l'auteur et la maison d'édition.

Les protestations du traducteur furent tellement véhémentes qu'on put difficilement ne pas en tenir compte. Le bon sens aurait voulu qu'on lui demandât alors de réparer luimême les ravages faits par l'intervention de l'editor. C'eût été trop contraire à la déontologie qui anime l'esprit d'editing, qui veut que la compétence de l'editor l'emporte sur celle du traducteur.

Comme on ne pouvait cependant pas nier complètement la pertinence des protestations du traducteur, on fit appel à un deuxième editor. On aurait pu s'attendre à ce que ce dernier prît contact avec le traducteur pour qu'à eux deux ils restaurent le manuscrit massacré par le premier. Au lieu de cela, que fit-il? Il adopta le parti inverse de celui de son prédécesseur et décida de supprimer tous les passés simples pour utiliser systématiquement le présent historique. On ne saura sans doute jamais quel raisonnement l'a mené à adopter ce parti, car il s'est bien gardé d'en informer le traducteur, dont il craignit sans doute une deuxième vague de protestations, et qu'il considérait vraisemblablement comme trop bête pour accéder à ce raisonnement... On peut toutefois imaginer que ce parti a été adopté sans grande méditation du texte lui-même, mais après un débat académique et abstrait sur la légitimité du présent historique par rapport au passé simple comme équivalent du prétérit anglais.

Quoi qu'il en soit, on peut imaginer les effets de l'application de cet esprit de système: d'une part, en raison du nombre considérable de formes verbales «corrigées», il s'en trouve forcément qui ont été oubliées; le texte est émaillé de passés simples qui font intrusion de manière incongrue au détour d'une phrase et qui ne concordent pas toujours avec les autres temps du paragraphe. D'autre part, les adaptations nécessaires pour faire concorder les autres temps sont souvent lourdes et maladroites, rompant le balancement des phrases. Le texte prend surtout une tonalité un peu étrange et artificielle que le lecteur percevra sans savoir à quoi l'attribuer, mais en sentant bien qu'il a affaire à du «langage traduction» et non à du français spontané.

Ce qui fait qu'un texte est «écrit» par rapport à un texte négligé, ou grossièrement mis en forme, tient parfois à des indices très ténus, des petites différences presque imperceptibles mais qui sont les plus intéressants à étudier dans le cadre d'une méthodologie de la traduction. Prenons-en un exemple : 
Version traducteur: (Amherst) s'attendait-il à se heurter à des troupes canadiennes remontant le cours du Richelieu ? Ou bien était-il si sûr que la tentative de Wolfe (...) se solderait par un échec qu'il n'osa pas s'avancer trop loin en territoire ennemi?

Et en effet, Wolfe est en situation délicate à la fin de l'été $1759 .$.

Après l'intervention du premier editor, l'attaque du deuxième paragraphe était devenue : En effet, Wolfe est en situation délicate à la fin de l'été 1759 (...) Le «et» rhétorique a été purement et simplement congédié, au prétexte sans doute que l'original anglais ne comportait pas de «and». Cet original dit en effet:

Certainly, Wolfe was in an awkward position (...)

Le gardien de la lettre du texte n'avait pas vu qu'«en effet» n'était pas équivalent à certainly, placé en tête de phrase en transition de deux paragraphes. Il n'avait pas senti la valeur rhétorique de ce dernier, à la fois confirmation emphatique d'une assertion et façon de relier deux paragraphes tout en dissociant leurs contenus. «En effet» ne remplit que la fonction sémantique de confirmation (qui aurait pu être aussi remplie par exemple par «il est bien vrai que»). Seule l'introduction de et remplit la fonction rhétorique remplie par la position de certainly.

On peut arguer que ce détail est de peu de poids dans le déroulement global du récit. C'est vrai. Aussi, le fait significatif n'est-il pas que le traducteur ait introduit cet élément, qui est en quelque sorte du surcroît de qualité. Ce qui est significatif, c'est le fait que l'editor ait jugé nécessaire de le gommer, comme il a gommé tous les tours rhétoriques français essayant de rendre le style du texte original.

Cette obstination à se priver des ressources offertes par la langue de traduction ne peut que laisser pantois !

Que conclure de ces quelques observations, dans le cadre d'un exposé aussi bref que celui-ci?

On ne s'arrêtera pas longuement à instruire le procès de gens à qui on fera le crédit de penser qu'ils manipulent les traductions en croyant bien faire. On soulignera seulement qu'il est étrange et regrettable que des gens dont le métier proclamé est l'amélioration de la communication et dont, en conséquence, la vocation devrait être d'aider les traducteurs à élargir leur palette de moyens d'expression, interviennent au contraire sur les textes en réduisant cette palette. Il ne s'agit pas ici d'accuser, mais de dégager les causes et les effets de cette intervention négative qui fait régresser une traduction arrivée à maturation vers un état de moindre accomplissement. Ces causes sont patentes. Elles ont déjà été énoncées plus haut: on pourrait dire qu'elles se ramènent toutes à une sorte de peur de quitter la terre ferme de la fidélité à la lettre pour celui de la fidélité à l'esprit. La manière de faire de ces manipulateurs de traduction fait penser aux noyés qui s'accrochent de toutes leurs forces au nageur qui veut les sauver, entravant ses mouvements au lieu de se laisser porter, et concourant ainsi à leur perte commune.

Cette crispation sur la lettre de son texte se nourrit chez l'auteur d'une crainte bien compréhensible de voir sa pensée trahie. Dans la divergence d'appréciation qui peut opposer le traducteur et l'editor, l'auteur sera tenté de faire confiance à l'editor, car ce dernier ne demande qu'à flatter son goût pour la littéralité et le calque. Son mode de travail - le phrase par phrase - s'y prête en effet éminemment. La littéralité réclamée par l'auteur lui épargne d'avoir à refaire tout le travail mental difficile opéré par le traducteur pour aboutir à une vraie traduction. À supposer même qu'il soit compétent - cela arrive - il est trop extérieur au texte pour s'intéresser à ce qui fait sa substance vive et le traite comme une chaîne de signes dont les constituants sont détricotés maille par maille. 
Ne tirons pas de conclusions abusivement généralisantes de l'analyse de ce cas d'espèce. Il n'y a pas d'un côté les traductions - toujours parfaites - produites par les traducteurs toujours compétents et de l'autre côté les «tripatouillages» effectués par des besogneux incompétents à la solde des éditeurs et des auteurs. Disons seulement que la tendance à dessaisir les traducteurs de leurs prérogatives au profit d'une caste de rédacteurs de tout poil porte en germe de graves dangers pour la qualité des traductions si ces rédacteurs ne sont pas recrutés sur des critères de compétence incontestable. Pour que leur apport soit bénéfique, il faudrait au moins qu'ils aient fait la preuve - comme les réviseurs - d'une compétence démontrée en traduction. Qui pourrait affirmer que tel est le cas aujourd'hui?

Notre étude n'aurait aucun intérêt de ce point de vue si le cas était unique. Malheureusement nombre de traductions subissent à des degrés divers le sort de celle examinée ici.

Du point de vue de la recherche sur la traduction, l'existence de ces procédures rédactionnelles n'est pas sans intérêt. Je n'ai pu ici que faire miroiter le parti qu'on pouvait en tirer, mais il est indéniable. De même que la pathologie permet au biologiste d'approfondir sa connaissance du fonctionnement normal du vivant, de même l'étude des processus mentaux qui défont le travail du traducteur peut nous apprendre beaucoup de choses sur les processus qui mènent aux traductions réussies.

J'aimerais, pour ma part, remettre entre les mains d'un jeune chercheur, pour qu'il l'exploite, le volumineux corpus dont j'ai entrouvert ici quelques feuillets...

\section{NOTES}

1. Habitant (en français du Canada) : agriculteur

2. Voyageurs (à l'époque dont il est question) : employés des compagnies de traite qui allaient commercer avec les Indiens à de grandes distances des centres urbains de la colonie.

3. Choix lexical lui-même surprenant, puisque manoir est le terme usuel pour désigner ce que l'auteur nomme, en anglais, manor house. 